Authors

Jun Ye, Tai Hyun Yoon, John L. Hall, Alan A. Madej, Jean-Marie Chartier, and Annick Chartier 


\title{
Accuracy Comparison of Absolute Optical Frequency Measurement between Harmonic-Generation Synthesis and a Frequency-Division Femtosecond Comb
}

\author{
Jun Ye, ${ }^{*}$ Tai Hyun Yoon, ${ }^{\dagger}$ and John L. Hall* \\ JILA, University of Colorado and National Institute of Standards and Technology, Boulder, Colorado 80309-0440 \\ Alan A. Madej, John E. Bernard, Klaus J. Siemsen, and Louis Marmet \\ Institute for National Measurement Standards, National Research Council, Ottawa, Canada KIA OR6 \\ Jean-Marie Chartier and Annick Chartier \\ Bureau International des Poids et Mesures, Pavillon de Breteuil, F-92312 Sèvres Cedex, France
}

(Received 12 June 2000)

\begin{abstract}
Using an iodine-stabilized He-Ne laser as a transfer oscillator, we compare absolute measurements of the optical frequency from a traditional frequency synthesis chain based on harmonic generation and from the frequency division technique of an ultrawide bandwidth femtosecond frequency comb. The agreement between these two measurements, both linked to the Cs standard, is $220 \pm 770 \mathrm{~Hz}$, yielding a measurement accuracy of $1.6 \times 10^{-12}$. We report $473612353604.8 \pm 1.2 \mathrm{kHz}$ as a preliminary updated value of the absolute frequency of the " $f$ " component for the He-Ne laser international standard at $633 \mathrm{~nm}$.
\end{abstract}

PACS numbers: 42.62.Eh, 06.20.Fn, 06.30.Bp

Optical frequency standards based on a single trapped ion or a collection of cold atoms have emerged as important tools in the test of fundamental physics. The unrivaled resolution, witnessed by single ion spectroscopy and its use in optical frequency standards [1] has allowed access to unprecedented stability and accuracy at the level of $10^{-16}$ for the operation frequency of a coherent oscillator. Recently, a spectroscopic linewidth below $10 \mathrm{~Hz}$ at a transition frequency of $10^{15} \mathrm{~Hz}$ has been reported [2]. A number of high quality optical frequency standards have already been demonstrated and their frequencies linked to the Cs microwave standard at various accuracy levels [3-5]. Recent developments in ultrawide bandwidth optical frequency combs based on femtosecond (fs) laser technology [6] have brought a revolutionary step in the field of optical frequency metrology promising further rapid advances in this field. An entire optical octave has been spanned by the comb and this has resulted in a single-step measurement process of absolute optical frequencies from microwave standards [7], a tremendous simplification from the traditional frequency chains based on the principle of harmonic synthesis. With such devices acting as the necessary frequency link of phase coherent transfer from the high to low frequencies, it now appears possible that atomic clocks based on optical transitions will become a reality.

A regularly spaced comb of optical frequencies is generated from a Kerr-lens mode-locked fs laser. The frequency spacing of the comb interval is equal to the inverse of the controlled cavity round-trip time of the laser. Initial studies have shown that the uniformity of the comb spacing reaches a level of better than $3 \times 10^{-17}$ [6]. To permit the coverage of an entire optical octave, the bandwidth of the comb emitted from the laser is further broadened by launching the pulse train into a microstructure fiber [8].
Precise optical frequency measurements have been carried out with this new technique [6,7]. However, during the course of measurements of several pre-existing optical standards, it was found that there were frequency offsets of a few $\mathrm{kHz}$ between the values measured using the comb technique and those measured previously with traditional frequency synthesis chains. To check the relative accuracy between these two approaches, and especially to establish confidence in the use of the straightforward and yet powerful fs-comb technique, it is important that an optical frequency standard be measured and compared using these two different methods at the same time. Recently we carried out just such a measurement, using a transportable iodine-stabilized $\mathrm{He}-\mathrm{Ne}$ laser $\left(\mathrm{HeNe} / \mathrm{I}_{2}\right)$ as a flywheel oscillator to transfer the optical frequency information between JILA and the National Research Council (NRC) of Canada. An absolute determination of the He-Ne laser frequency was obtained at each location using the two different techniques. At JILA, the He-Ne laser frequency was measured using a fs-comb system, while at NRC the measurement was carried out with a traditional Cs based frequency synthesis chain [4]. The agreement between these two measurements is $220 \pm 770 \mathrm{~Hz}$, i.e., an (in-)accuracy of $1.6 \times 10^{-12}$ of the optical frequency at $474 \mathrm{THz}$. This intercomparison work has also resulted in a correction to the accepted value of the absolute frequency of the international length standard, the $\mathrm{HeNe} / \mathrm{I}_{2}$ laser at $633 \mathrm{~nm}$, as monitored at the Bureau International des Poids et Mesures (BIPM). The work represents the first such accuracy check between two independent laboratories using different techniques for optical frequency measurement and should prove to be useful for future progress.

The frequency stability and reproducibility of the transportable $\mathrm{HeNe} / \mathrm{I}_{2}$ laser (JILA-145) is of critical 
importance since the absolute frequency intercomparison is carried out with this He-Ne laser as the common measurement target. The operation parameters of JILA-145 were strictly observed and maintained throughout the intercomparison [9]. Also, the laser frequency before and after transportation was measured with heterodyne beat against another continuously running $\mathrm{He}-\mathrm{Ne}$ laser (NIST-126). Heterodyne beat measurements between the two He-Ne lasers allowed accurate calibration of the laser frequency shift vs operation parameters, which were then used to correct the observed laser frequency to the value the laser would produce at the nominal operating conditions. Four hyperfine components, namely, $g\left(a_{15}\right)$, $f\left(a_{16}\right), e\left(a_{17}\right)$, and $d\left(a_{18}\right)$ of the 11-5 R(127) transition of ${ }^{127} \mathrm{I}_{2}$ molecules, are used in typical beat measurements. When locked at each of these hyperfine resonances, we found that both of our lasers gave highly reproducible frequency output $(\sim 250 \mathrm{~Hz})$ if the laser operation parameters were maintained at constant values or corrections were applied using the measured frequency-shift coefficients. In fact, the frequency of JILA-145 was measured to be within $200 \mathrm{~Hz}$ before and after the frequency intercomparison round-trip JILA $\rightarrow$ NRC $\rightarrow$ JILA.

Figure 1(a) shows the experimental schematic of the absolute frequency measurement of the JILA-145 laser based

(a)

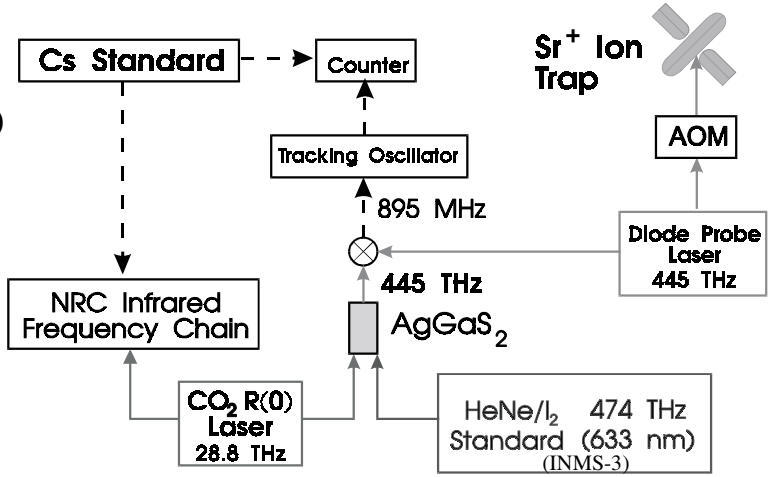

(b)

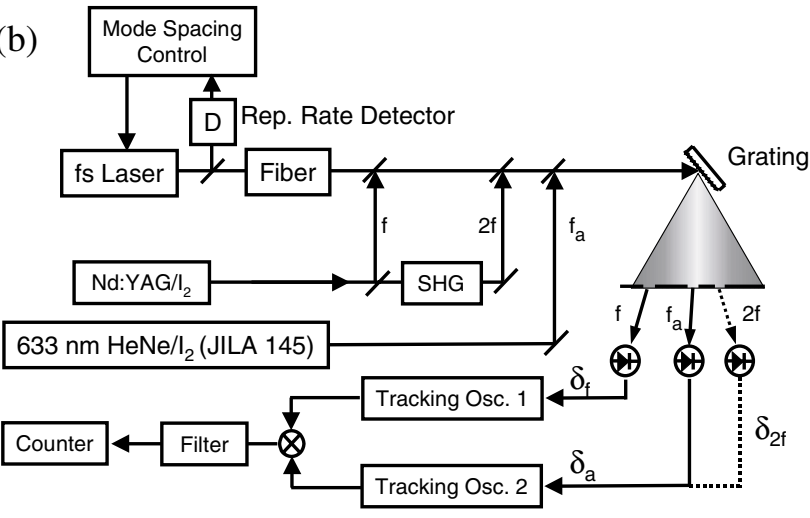

FIG. 1. (a) The NRC Cs based optical frequency chain used to measure the frequency of $\mathrm{HeNe} / \mathrm{I}_{2}$ relative to the single $\mathrm{Sr}^{+}$ frequency standard. (b) The JILA fs-comb setup used to measure the absolute frequencies of iodine stabilized $\mathrm{Nd}$ :YAG and $\mathrm{He}-\mathrm{Ne}$ lasers. on the NRC frequency synthesis chain. Here a $\mathrm{CO}_{2}$ laser operating on the $R(0)$ laser transition and stabilized on the $Q(15)$ reference absorption transition in $\mathrm{OsO}_{4}$ at $28.8 \mathrm{THz}$ [10] was linked to the Cs standard [11]. Another reference laser input to the measurement was the frequency of a laser stabilized to the electric quadrupole allowed $5 s^{2} S_{1 / 2} \rightarrow 4 d^{2} D_{5 / 2}$ transition at $445 \mathrm{THz}$ in the single trapped and laser cooled ${ }^{88} \mathrm{Sr}^{+}$ion. The absolute value of the transition frequency was determined with a similar chain a year ago [4]. The difference frequency between the He-Ne laser at $474 \mathrm{THz}$ and the $\mathrm{CO}_{2}$ laser was generated in a $\mathrm{AgGaS}_{2}$ optical crystal and the beat between this generated frequency and that of the $445 \mathrm{THz}$ single ion stabilized laser was obtained with an offset of $895 \mathrm{MHz}$. The beat frequency was counted with the aid of a phase-locked tracking oscillator. With sufficient averaging, the measurement accuracy was primarily limited to the present uncertainty $(200 \mathrm{~Hz})$ of the $445 \mathrm{THz} \mathrm{Sr}{ }^{+}$frequency standard. The uncertainty of the $28.8 \mathrm{THz}\left(\mathrm{CO}_{2}\right)$ standard decreases below $20 \mathrm{~Hz}$ at an averaging time of $100 \mathrm{~s}$. The absolute frequency of a $\mathrm{HeNe} / \mathrm{I}_{2}$ laser of NRC (INMS-3) was measured in the chain. The beat frequency between INMS-3 and JILA-145 was recorded before and after the chain measurement, therefore establishing the absolute frequency of JILA-145 with reference to the NRC chain. A portable He-Ne laser from BIPM, BIPM-P3, also participated in the intercomparison and its frequency was measured relative to both JILA-145 and INMS-3. The frequency difference between BIPM-P3 and BIPM-4, which serves as the representative value for a practical realization of the definition of the meter [12], was measured at BIPM before and after this intercomparison. Hence the frequency of the transfer oscillator, JILA-145, is now connected to the BIPM standard, as well as to both chains in NRC and JILA.

Figure 1(b) illustrates the JILA frequency measurement using the fs comb. The comb spans an entire optical octave $(>300 \mathrm{THz})$, leading to a direct measurement of an optical frequency relative to a microwave standard when we phase coherently bridge the gap between the fundamental and its second harmonic [7]. The comb spacing of $100 \mathrm{MHz}$, corresponding to the laser pulse repetition rate, is phase locked to a stable radio frequency reference, which is itself referenced to the NIST ensemble of Cs clocks via common view Global Positioning System reception. In this experiment, an ultrastable $\mathrm{cw}$ laser serves to establish the absolute frequencies of the whole comb. The cw laser is a Nd:YAG laser at $1064 \mathrm{~nm}$ along with its second harmonic at $532 \mathrm{~nm}$. The Nd:YAG laser is stabilized to the iodine hyperfine component $a_{10}$ of the $R(56) 32-0$ transition at $532 \mathrm{~nm}$, with a frequency stability of $5 \times 10^{-14}$ at $1 \mathrm{~s}$ averaging time and reaching $4 \times 10^{-15}$ at $700 \mathrm{~s}$ [13]. The frequency of the stabilized Nd:YAG laser at $1064 \mathrm{~nm}$ is determined by the measurement of the frequency gap between $1064 \mathrm{~nm}$ and its second harmonic at $532 \mathrm{~nm}$ in terms of the comb spacing. We have thus established an 
accurate frequency grid spanning the whole near-IR and visible spectrum and the He-Ne laser frequency can be easily measured using this frequency comb. Our approach, as shown in Fig. 1(b), is to count simultaneously the two beat frequencies, one between JILA-145 and its corresponding comb line and the other between the Nd:YAG laser and one of its neighboring comb lines. The typical signal-to-noise ratio $(\mathrm{S} / \mathrm{N})$ of the beat at 1064,633 , or $532 \mathrm{~nm}$ is $\sim 20 \mathrm{~dB}$ in a $100 \mathrm{kHz}$ bandwidth. This $\mathrm{S} / \mathrm{N}$ permits robust phase locking between the beat signals and the tracking oscillators, leading to an accurate frequency counting. Prior to the measurement of the frequency gap between $\mathrm{He}-\mathrm{Ne}$ and $\mathrm{Nd}$ :YAG lasers, the absolute frequency of the Nd:YAG laser is always measured first, with a typical standard deviation of about $200 \mathrm{~Hz}$ between different measurements.

To have confidence in the measurement results from these frequency chains, it is important to study the stability of the counted frequencies. The final measurement should be limited by the frequency stability of the standard under investigation. In Fig. 2 we summarize the stability results from both chains. Figure 2(a) shows the fractional instability (expressed in terms of the Allan standard deviation) of the NRC chain measurement of INMS-3 (in filled circles). An overlaid curve in open squares indicates the frequency stability of INMS-3 measured independently
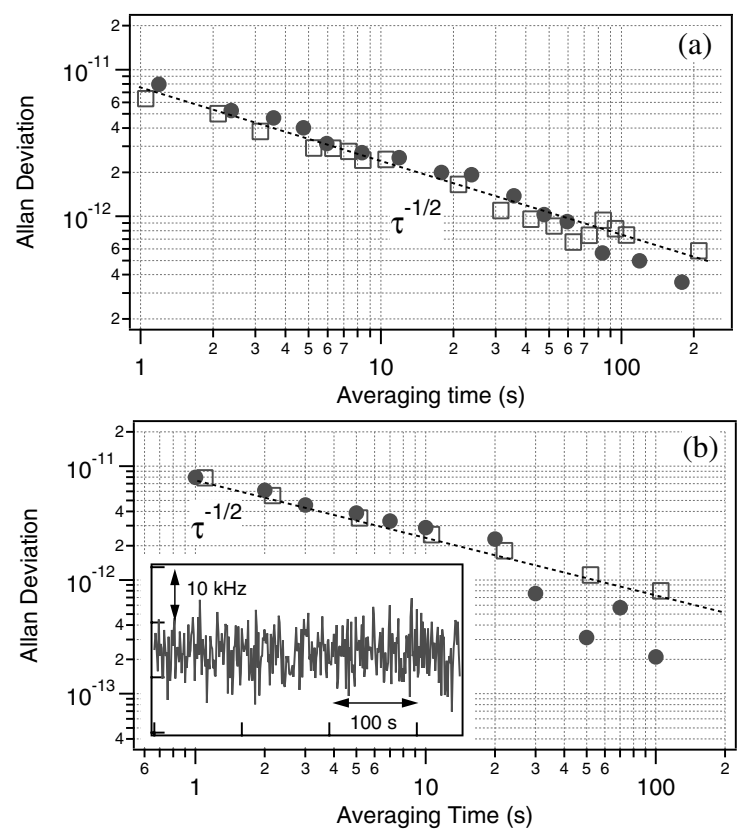

FIG. 2. Stability of frequency measurement in both NRC and JILA frequency chains. (a) Allan standard deviations of the NRC laser, INMS-3 (in open square $\square$ ), and of the frequency measurement of the same laser in NRC chain (in filled circles, -). (b) Allan deviation (in filled circles, ) of the beat frequency between JILA-145 and the Nd:YAG laser in the JILA fs-comb system (time record shown in the inset). Independently measured Allan deviation of JILA-145 is also shown (in open square, $\square$ ). The $\tau^{-1 / 2}$ decrease of the Allan deviation (associated with white frequency noise) is shown in both (a) and (b) in dotted lines. outside of the chain. Clearly the laser instability is the dominant contribution to the noise of the chain measurement. This conclusion holds true for the JILA fs-comb measurement system as well. The inset in Fig. 2(b) shows a typical record of the beat frequency as a function of time with an averaging (gate) time of $1 \mathrm{~s}$. This measured frequency corresponds to the sum or difference between the two beat frequencies (1064 nm vs comb and $633 \mathrm{~nm}$ vs comb) as explained above. The corresponding Allan deviation is shown in Fig. 2(b), with the fs-comb measurement result displayed in filled circles and the data associated with JILA-145 measured in an independent beat experiment (open squares). It thus appears that the measurement noise of the fs-comb system is below the intrinsic noise level of the $\mathrm{HeNe} / \mathrm{I}_{2}$. However, the smaller values of Allan deviation associated with both frequency measurements at long averaging times [ $>80 \mathrm{~s}$ for (a) and $>30 \mathrm{~s}$ for (b)] result from poor statistics of a few measurement points. In addition, owing to the superior stability displayed by the Nd:YAG laser system (200 times more stable than the $\mathrm{He}-\mathrm{Ne}$ system), we could clearly observe the additional noise contributed by the fs-comb system when the absolute frequency of Nd:YAG was measured. We now have direct evidence that our fs-comb system is currently limited by the stability of the rf oscillator we use to stabilize the comb spacing [14].

The absolute frequency of the JILA-145 laser measured in both NRC and JILA chains is summarized in Fig. 3. The fs-comb measurement at JILA gave a value of $473612353602.26 \pm 0.34 \mathrm{kHz}$ for the frequency of JILA-145 locked to the " $f$ " component of the ${ }^{127} \mathrm{I}_{2}$ 11-5 $R(127)$ transition. The experimental uncertainty of $340 \mathrm{~Hz}$ is consistent with the contributions from the $\mathrm{Nd}$ :YAG frequency measurement $(200 \mathrm{~Hz}), \mathrm{He}-\mathrm{Ne}$ laser $(250 \mathrm{~Hz})$, and the rf oscillator $(100 \mathrm{~Hz})$. The NRC chain determined the absolute frequency of INMS-3 to be $473612353595.37 \pm 0.25 \mathrm{kHz}$ when the laser was also locked to the $f$ component. The intercomparison between JILA-145 and INMS-3 revealed a frequency difference of $+6.4 \pm 0.6 \mathrm{kHz}$. The $0.6 \mathrm{kHz}$ uncertainty quoted here results from the fact that four hyperfine components

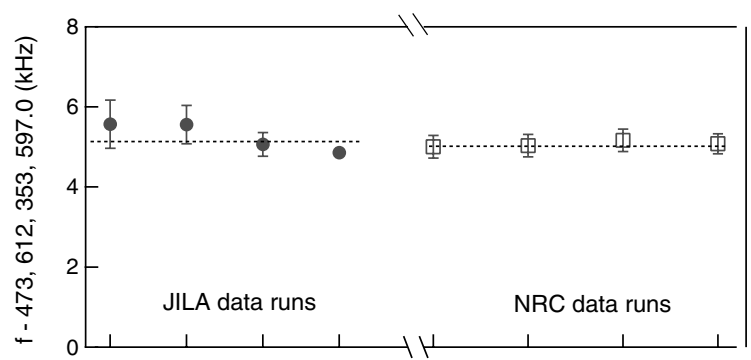

FIG. 3. The frequency of the transfer oscillator, JILA-145, as determined from both chains. Their difference in the measured frequency is $220 \pm 770 \mathrm{~Hz}$. The frequency scale has been plotted relative to the current recommended value for the " $f$ " hyperfine component [12] 
(" $d$, " " $e, "$ " $f, "$ " $g$ ") were used in the heterodyne beat between the two He-Ne lasers. The reproducibility of the laser locked on a particular component, for example, $f$, is about $0.25 \mathrm{kHz}$. The frequency of JILA-145 was shifted by $+0.27 \pm 0.15 \mathrm{kHz}$ when it was returned to JILA from NRC due to a slight decrease of the iodine-cell temperature $(\sim 20 \mathrm{mK})$. Adding the three numbers (INMS-3 frequency, frequency difference of $+6.4 \mathrm{kHz}$, and frequency shift of $+0.27 \mathrm{kHz}$ ) together leads to an absolute frequency $473612353602.04 \pm 0.69 \mathrm{kHz}$ for JILA-145, as determined from the NRC chain. Therefore the difference in the measured frequency of JILA-145 between the two chains is $220 \pm 770 \mathrm{~Hz}$, limited by the uncertainties of the $\mathrm{He}-\mathrm{Ne}$ laser intercomparison $(\sim 620 \mathrm{~Hz})$, of the JILA fs-comb chain $(\sim 340 \mathrm{~Hz})$, and of the ion reference frequency in the NRC chain $(\sim 200 \mathrm{~Hz})$.

The frequency of the BIPM-4 standard is linked to both chains through the transportable BIPM-P3 laser. The frequency differences between the transfer oscillators, namely, between JILA-145 and BIPM-P3, and between INMS-3 and BIPM-P3, were repeatedly measured at NRC during the intercomparison, with a typical standard deviation of $\sim 0.7 \mathrm{kHz}$ for the heterodyne beat measurements. The absolute frequency of BIPM- 4 can thus be established through both channels and be compared. The NRC chain gave the BIPM-4 frequency at $473612353604.4 \pm 1.4 \mathrm{kHz}$ while the JILA measurement gave $473612353605.2 \pm 1.9 \mathrm{kHz}$. Both results are higher than the CIPM recommended [12] value for the $f$ hyperfine component, with the NRC data higher by $7.4 \mathrm{kHz}$ and the JILA data higher by $8.2 \mathrm{kHz}$. Figure 4 summarizes the historical measurement results of the $\mathrm{HeNe} / \mathrm{I}_{2}$ frequency with respect to our current measurement. Direct frequency measurement was first reported by a group at NBS (now NIST) in 1983 [15] without a direct link to the Cs clock. A joint frequency measurement of a $\mathrm{HeNe} / \mathrm{I}_{2}$ laser was performed at the Laboratoire Primaire du Temps et des Fréquences in Paris, leading to an improved value of the Rydberg constant [16]. The work reported here not only improves the measurement precision but also gives a more consistent picture of the actual frequency of the $633 \mathrm{~nm}$ standard.

In summary, we have performed a comparison of absolute optical frequency measurements between a traditional frequency synthesis chain and a femtosecond frequency comb system. The agreement between these two systems, both linked to the Cs lock, is $220 \pm 770 \mathrm{~Hz}$, i.e., an (in-) accuracy of $1.6 \times 10^{-12}$. We report $473612353604.8 \pm$ $1.2 \mathrm{kHz}$ as a preliminary updated value of the absolute frequency of the $f$ component for the He-Ne laser international standard at $633 \mathrm{~nm}$.

We thank S. Diddams, S. T. Cundiff (JILA), and P. Dubé (NRC) for useful contributions. The work at JILA is sup-

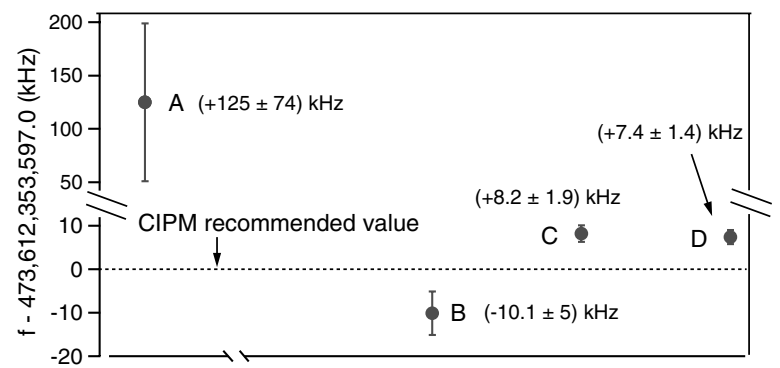

FIG. 4. Absolute frequency measurement of the He-Ne laser stabilized on the hyperfine component $f$ of the ${ }^{127} \mathrm{I}_{2} 11-5 R(127)$ transition. A: NBS 1983 (Ref. [15]), B: Paris 1993 (Ref. [16]), $C$ : JILA 2000 (this work), and D: NRC 2000 (this work).

ported by NIST, NASA, and NSF. The work at NRC is supported by NRC, Canada and the Canadian Institute for Photonic Innovations (CIPI). T. H. Y. acknowledges financial support from JILA.

*Quantum Physics Division, NIST, Boulder, CO.

Electronic address: ye@jila.colorado.edu

†Permanent address: KRISS, 1 Toryong, Yusong, Taejon 305-600, Korea.

[1] A. A. Madej and J. E. Bernard, in Frequency Measurement and Control: Advanced Techniques and Future Trends, Springer Topics in Applied Physics Vol. 79, edited by A. N. Luiten (Springer, Berlin, 2000).

[2] R. J. Rafac, B.C. Young, J. A. Beall, W. M. Itano, D. J. Wineland, and J.C. Bergquist, Phys. Rev. Lett. 85, 2462 (2000).

[3] H. Schnatz et al., Phys. Rev. Lett. 76, 18 (1996).

[4] J. E. Bernard et al., Phys. Rev. Lett. 82, 3228 (1999).

[5] C. Schwob et al., Phys. Rev. Lett. 82, 4960 (1999).

[6] Th. Udem et al., Phys. Rev. Lett. 82, 3568 (1999); Th. Udem et al., Opt. Lett. 24, 881 (1999).

[7] S. A. Diddams et al., Phys. Rev. Lett. 84, 5102 (2000); D. J. Jones, Science 288, 635 (2000).

[8] J. Ranka et al., Opt. Lett. 25, 25 (2000).

[9] T. H. Yoon et al. (to be published).

[10] A. A. Madej et al., IEEE Trans. Instrum. Meas. 48, 553 (1999).

[11] B. G. Whitford, Metrologia 30, 145 (1993).

[12] T. J. Quinn, Metrologia 36, 211 (1999).

[13] J. L. Hall et al., IEEE Trans. Instrum. Meas. 48, 583 (1999); J. Ye et al., ibid. 48, 544 (1999).

[14] J. Ye and J. L. Hall (to be published).

[15] D. A. Jennings et al., Opt. Lett. 8, 136 (1983). We converted their measured data on " $i$ " to " $f$," using the standard interval value. [See Refs. [6] and [9] in P. Zhao et al., Phys. Rev. A 39, 2888 (1989).]

[16] O. Acef et al., Opt. Commun. 97, 29 (1993); F. Nez et al., Phys. Rev. Lett. 69, 2326 (1992). 